\title{
Chandra X-ray Observations of 12 Millisecond Pulsars in the Globular Cluster M28
}

\section{Citation}

Bogdanov, Slavko, Maureen van den Berg, Mathieu Servillat, Craig O. Heinke, Jonathan E. Grindlay, Ingrid H. Stairs, Scott M. Ransom, Paulo C. C. Freire, Steve Bégin, and Werner Becker. 2011. "CHANDRAX-RAY OBSERVATIONS OF 12 MILLISECOND PULSARS IN THE GLOBULAR CLUSTER M28." The Astrophysical Journal 730 (2): 81. https:// doi.org/10.1088/0004-637x/730/2/81.

\section{Permanent link}

http://nrs.harvard.edu/urn-3:HUL.InstRepos:41399768

\section{Terms of Use}

This article was downloaded from Harvard University's DASH repository, and is made available under the terms and conditions applicable to Other Posted Material, as set forth at http:// nrs.harvard.edu/urn-3:HUL.InstRepos:dash.current.terms-of-use\#LAA

\section{Share Your Story}

The Harvard community has made this article openly available.

Please share how this access benefits you. Submit a story.

\section{Accessibility}




\title{
CHANDRA X-RAY OBSERVATIONS OF 12 MILLISECOND PULSARS IN THE GLOBULAR CLUSTER M28
}

\author{
Slavko Bogdanov ${ }^{1,9}$, Maureen van den Berg $^{2}$, Mathieu Servillat ${ }^{2}$, Craig O. Heinke ${ }^{3}$, Jonathan E. Grindlay ${ }^{2}$, \\ Ingrid H. Stairs ${ }^{4}$, Scott M. Ransom ${ }^{5}$, Paulo C. C. Freire ${ }^{6}$, Steve Bégin ${ }^{4}, 7$ and Werner Becker ${ }^{8}$ \\ ${ }^{1}$ Department of Physics, McGill University, 3600 University Street, Montreal, QC H3A 2T8, Canada; bogdanov@ physics.mcgill.ca \\ 2 Harvard-Smithsonian Center for Astrophysics, 60 Garden Street, Cambridge, MA 02138, USA \\ ${ }^{3}$ Department of Physics, University of Alberta, 1132289 Avenue, Edmonton, AB T6G 2G7, Canada \\ ${ }^{4}$ Department of Physics, University of British Columbia, 6224 Agricultural Road Vancouver, BC V6T 1Z1, Canada \\ ${ }^{5}$ National Radio Astronomy Observatory, 520 Edgemont Road, Charlottesville, VA 22901, USA \\ ${ }^{6}$ Max-Planck-Institut für Radio Astronomie, Auf dem Hügel 69, D-53121 Bonn, Germany \\ ${ }^{7}$ Département de physique, de génie physique et d'optique, Université Laval, Quebec, QC G1K 7P4, Canada \\ ${ }^{8}$ Max-Planck-Institut für Extraterrestrische Physik, D-85740 Garching bei München, Germany \\ Received 2010 April 19; accepted 2011 January 24; published 2011 March 8
}

\begin{abstract}
We present a Chandra X-ray Observatory investigation of the millisecond pulsars in the globular cluster M28 (NGC 6626). In what is one of the deepest X-ray observations of a globular cluster, we firmly detect seven and possibly detect two of the 12 known M28 pulsars. With the exception of PSRs B1821-24 and J1824-2452H, the detected pulsars have relatively soft spectra, with X-ray luminosities $10^{30}-10^{31} \mathrm{erg} \mathrm{s}^{-1}(0.3-8 \mathrm{keV})$, similar to most "recycled" pulsars in 47 Tucanae and the field of the Galaxy, implying thermal emission from the pulsar magnetic polar caps. We present the most detailed X-ray spectrum to date of the energetic PSR B1821-24. It is well described by a purely non-thermal spectrum with spectral photon index $\Gamma=1.23$ and luminosity $1.4 \times 10^{33} \Theta$ $(D / 5.5 \mathrm{kpc})^{2} \mathrm{erg} \mathrm{s}^{-1}(0.3-8 \mathrm{keV})$, where $\Theta$ is the fraction of the sky covered by the $\mathrm{X}$-ray emission beam $(\mathrm{s})$. We find no evidence for the previously reported line emission feature around $3.3 \mathrm{keV}$, most likely as a consequence of improvements in instrument calibration. The X-ray spectrum and pulse profile of PSR B1821-24 suggest that the bulk of unpulsed emission from this pulsar is not of thermal origin, and is likely due to low-level non-thermal magnetospheric radiation, an unresolved pulsar wind nebula, and/or small-angle scattering of the pulsed X-rays by interstellar dust grains. The peculiar binary PSR J1824-2452H shows a relatively hard X-ray spectrum and possible variability at the binary period, indicative of an intrabinary shock formed by interaction between the relativistic pulsar wind and matter from its non-degenerate companion star.
\end{abstract}

Key words: globular clusters: general - globular clusters: individual (NGC 6626) - pulsars: general - pulsars: individual (PSR B1821-24, PSR J1824-2452H) - stars: neutron - X-rays: stars

Online-only material: animation, color figures

\section{INTRODUCTION}

Rotation-powered millisecond pulsars (MSPs) are faint X-ray sources, with typical luminosities of $L_{X} \sim 4 \times 10^{30}-10^{31} \mathrm{erg} \mathrm{s}^{-1}$ and up to $\sim 10^{33} \mathrm{erg} \mathrm{s}^{-1}$. Due to the long exposures required to study MSPs in detail, globular clusters provide a particularly efficient way to observe them in large numbers. This has only become feasible in the past decade, owing to the subarcsecond angular resolution of the Chandra X-Ray Observatory that is essential for individually resolving the numerous $\mathrm{X}$-ray sources in the dense cores of clusters. Thus far, a total of $\sim 30$ X-ray counterparts of MSPs have been identified with Chandra in globular clusters, including 47 Tuc (Grindlay et al. 2002; Bogdanov et al. 2006), NGC 6397 (Grindlay et al. 2002; Bogdanov et al. 2010), M28 (Becker et al. 2003), M4 (Bassa et al. 2004), NGC 6752 (D'Amico et al. 2002), M71 (Elsner et al. 2008), and Terzan 5 (Heinke et al. 2006). This appreciable sample has already provided important insight into the general $\mathrm{X}$-ray properties of the MSP population in the Galaxy.

The globular cluster Messier 28 (NGC 6626) is at a distance of $D \approx 5.5 \mathrm{kpc}$. The reddening toward M28 $E(B-V)=0.43$ (Harris 1996) implies a hydrogen column density of $N_{\mathrm{H}} \approx$ $2.4 \times 10^{21} \mathrm{~cm}^{-2}$. For over two decades, M28 was known to host a single MSP, PSR B1821-24 (Lyne et al. 1987). Recently, the

\footnotetext{
9 Canadian Institute for Advanced Research Junior Fellow.
}

Green Bank Telescope detected 11 additional radio pulsars in M28 (Bégin 2006) making it the cluster with the third largest number of known pulsars to date after Terzan 5 (at least 33; see Ransom et al. 2005) and 47 Tucanae (23; see Camilo et al. 2000; Freire et al. 2003, and references therein). ${ }^{10}$ These new pulsar discoveries make M28 a well-suited globular cluster for effective X-ray studies of a sizable population of MSPs.

Becker et al. (2003) have previously reported on a set of $\sim 40.9$ ks Chandra X-ray Observatory ACIS-S observations of M28 (ObsIDs 2683, 2684, 2685) which probed down to a luminosity of $L_{X} \sim 6 \times 10^{30} \mathrm{erg} \mathrm{s}^{-1}$ (assuming a source detection threshold of $\sim 10$ counts) and detected 46 sources, only 12 of which are within one core radius $(0.24)$ and 26 within the half-mass radius (1'56). Due to the relatively short exposure, just six sources had sufficient counts for a spectral fitting analysis, which included the original globular cluster MSP, PSR B1821-24. Rutledge et al. (2004) have used the superb timing capabilities of the High Resolution Camera (HRC)-S instrument on board Chandra to study the X-ray pulsations from B1821-24. More recently, this MSP was again targeted with HRC-S to calibrate the absolute timing precision of this detector (unpublished data).

In this paper, we present new deep Chandra X-ray Observatory ACIS-S observations of M28 and its 12 known

\footnotetext{
${ }^{10}$ For an up-to-date list of all known globular cluster MSPs see http://www.naic.edu/ pfreire/GCpsr.html.
} 
Table 1

Chandra Observations of M28

\begin{tabular}{llcc}
\hline \hline $\begin{array}{l}\text { Telescope/ } \\
\text { Instrument }\end{array}$ & $\begin{array}{c}\text { Epoch of } \\
\text { Observation }\end{array}$ & $\begin{array}{c}\text { Observation } \\
\text { ID }\end{array}$ & $\begin{array}{c}\text { Exposure } \\
\text { Time (ks) }\end{array}$ \\
\hline Chandra/ACIS-S & 2002 Jul 4 & 2684 & 12.9 \\
Chandra/ACIS-S & 2002 Aug 4 & 2685 & 13.7 \\
Chandra/ACIS-S & 2002 Sep 9 & 2683 & 14.3 \\
Chandra/HRC-S & 2002 Nov 8 & 2797 & 49.4 \\
Chandra/HRC-S & 2006 May 27 & 6769 & 41.1 \\
Chandra/ACIS-S & 2008 Aug 7 & 9132 & 144.1 \\
Chandra/ACIS-S & 2008 Aug 10 & 9133 & 55.2 \\
\hline
\end{tabular}

pulsars. This investigation sheds further light onto the general X-ray properties of the Galactic population of MSPs. A comprehensive analysis of the other X-ray source classes in M28 will be presented elsewhere (M. van den Berg et al. 2011, in preparation). The present work is outlined as follows. In Section 2, we describe the observations and data reduction and analysis procedures. In Section 3, we present the imaging analysis and detection methods of the X-ray counterparts to the MSPs. In Section 4 we conduct a spectroscopic and timing study of PSR B1821-24 (M28 A), while in Section 5 we focus on the peculiar binary PSR J1824-2452H (M28 H). In Section 6, we discuss the remaining pulsars in the cluster. We offer conclusions in Section 7.

\section{OBSERVATION AND DATA REDUCTION}

The new Chandra data set presented herein was acquired during two separate observations in Cycle 9, on 2008 August 7 (ObsID 9132) and 2008 August 10 (ObsID 9133) for 144.1 and $55.2 \mathrm{ks}$, respectively. For both exposures, the optical center of the cluster at $\alpha=18^{\mathrm{h}} 24^{\mathrm{m}} 32^{\mathrm{s}} .89$ and $\delta=-24^{\circ} 52^{\prime} 11^{\prime \prime} .4$ (Shawl $\&$ White 1986) was positioned $1^{\prime}$ off-axis from the nominal aim point of the back-illuminated ACIS-S3 CCD, configured in VFAINT telemetry mode. We also include the three Cycle 3 ACIS-S observations of 14.3, 12.9, and 13.7 ks (ObsIDs 2683, 2684, and 2685, respectively) from Becker et al. (2003), also taken in VFAINT mode. In the analysis of PSR B1821-24 (see Section 4), we make use of the two HRC-S exposures of 49.4 and $41.1 \mathrm{ks}$ (ObsIDs 2797 and 6769, respectively) in the special SI mode, which permits a timing resolution of $\sim 16 \mu \mathrm{s}$. All observations used herein are summarized in Table 1.

The data re-processing, reduction, and analysis were performed using $\mathrm{CIAO}^{11}$ 4.1.2. Starting from the level 1 data products of the ACIS-S observations, we first removed pixel randomization from the standard pipeline processing to aid in source disentanglement in the crowded cluster core. In addition, for the purposes of faint source detection we applied the background cleaning algorithm specific to the VFAINT telemetry mode. However, this procedure tends to reject real source counts for relatively bright sources. Consequently, the backgroundcleaned data were not used for the spectral analyses discussed in Sections 4 and 5. Before co-adding the five ACIS-S images, the individual aspect solutions were reprojected relative to the longest observation (ObsID 9132) using the brightest sources in the cluster to correct for any differences in the absolute astrometry between the individual observations. The summed ACIS-S data result in a $237.1 \mathrm{ks}$ effective exposure time, one of the deepest X-ray observations of a globular cluster, with a limiting sensitivity of $L_{X} \sim 1 \times 10^{30} \mathrm{erg} \mathrm{s}^{-1}$ for a $1 \mathrm{keV}$ source

\footnotetext{
11 Chandra Interactive Analysis of Observations, available at
} http://cxc.harvard.edu/ciao/ at the distance of $D=5.5 \mathrm{kpc}$ and hydrogen column density $N_{\mathrm{H}}=2.4 \times 10^{21} \mathrm{~cm}^{-2}$.

For the spectroscopy and variability analyses of the X-ray counterparts to the MSPs, we extracted the emission from polygonal (roughly circular) regions that enclose $\sim 90 \%$ of the total source energy at $1.5 \mathrm{keV}$. To permit spectral fitting in XSPEC, ${ }^{12}$ the extracted source counts in the $0.3-8 \mathrm{keV}$ range were grouped in energy bins so as to ensure at least 15 counts per bin. The background was taken from three source-free regions in the image around the cluster core. For the pulse profile of pulsar $A$ and variability analysis for pulsar $H$, the photon arrival times were reduced to the solar system barycenter, using the CIAO tool axbary assuming the DE405 JPL solar system ephemeris.

\section{IMAGING ANALYSIS AND SOURCE DETECTION}

Figure 1 shows the co-added image of all ACIS-S exposures (237.1 ks in total) of the core of M28 in the $0.5-2 \mathrm{keV}$ band (where most of the emission from MSPs is expected), with $1^{\prime \prime}$ circles centered on the 12 current best radio pulsar timing positions (S. Bégin et al. 2011, in preparation). It is apparent that the unresolved diffuse emission in the shallower ACIS-S observations reported by Becker et al. (2003) is mostly resolved into multiple faint sources, with some apparently coinciding with the known radio pulsars in M28. To formally identify the X-ray counterparts to the pulsars, we used the CIAO tool wavdetect, which correlates the image with Mexican-hat wavelets of various scales to identify sources. We also used the PWDetect ${ }^{13}$ script (Damiani et al. 1997a, 1997b), which is generally more effective at identifying faint sources near much brighter sources. Subsequently, we employed the IDL script acis_extract ${ }^{14}$ (Broos et al. 2010) to confirm the validity of the source detections reported by wavdetect and PWDetect and refine the source positions.

For PSR B1821-24 (henceforth MSP A, M28 A, or pulsar A), we find an offset between the radio pulsar position from Ray et al. (2008) and the known X-ray counterpart of $\Delta \alpha=$ $-0^{\prime \prime} .15$ and $\Delta \delta=-0^{\prime \prime} .26$ in right ascension and declination, respectively. Thus, we apply this correction to the astrometric frame of the X-ray data set, which results in significantly better matches between most of the radio MSPs and X-ray sources. The positional uncertainties of the detected X-ray sources were computed using the empirical relation from Hong et al. (2005), which yields the $95 \%$ error radius, $r_{95 \%}$. We note that the uncertainties in the radio timing positions for the M28 pulsars are generally $\lesssim 0$ ' 1 . Therefore, the dominant source of uncertainty in the X-ray counterpart matches is the error in the X-ray source position, with the exception of pulsars D and I. Pulsar D is significantly younger (based on its characteristic age $\tau \equiv \dot{P} / 2 P \approx 26 \mathrm{Myr}$ ) than the other pulsars in M28 and has a comparatively long spin period of $P=79 \mathrm{~ms}$. As such, it exhibits more timing noise, resulting in an uncertainty in its radio position, especially in declination, comparable to $r_{95 \%}$ $\left(\sim 0.5-1^{\prime \prime}\right)$. The error ellipse shown in Figure 1 was obtained by doubling the formal uncertainties in the pulsar position obtained with TEMPO. In the case of MSP I, the presence of random and irregular radio eclipses, apparent long-term variations in the orbital motion, and infrequent detections of the pulsar pose major difficulties in deriving a unique phase-connected

\footnotetext{
12 Available at http://heasarc.nasa.gov/docs/xanadu/xspec/index.html.

13 See http://www.astropa.unipa.it/progetti_ricerca/PWDetect/ for details.

14 Available at http://www.astro.psu.edu/xray/docs/TARA/

ae_users_guide.html.
} 


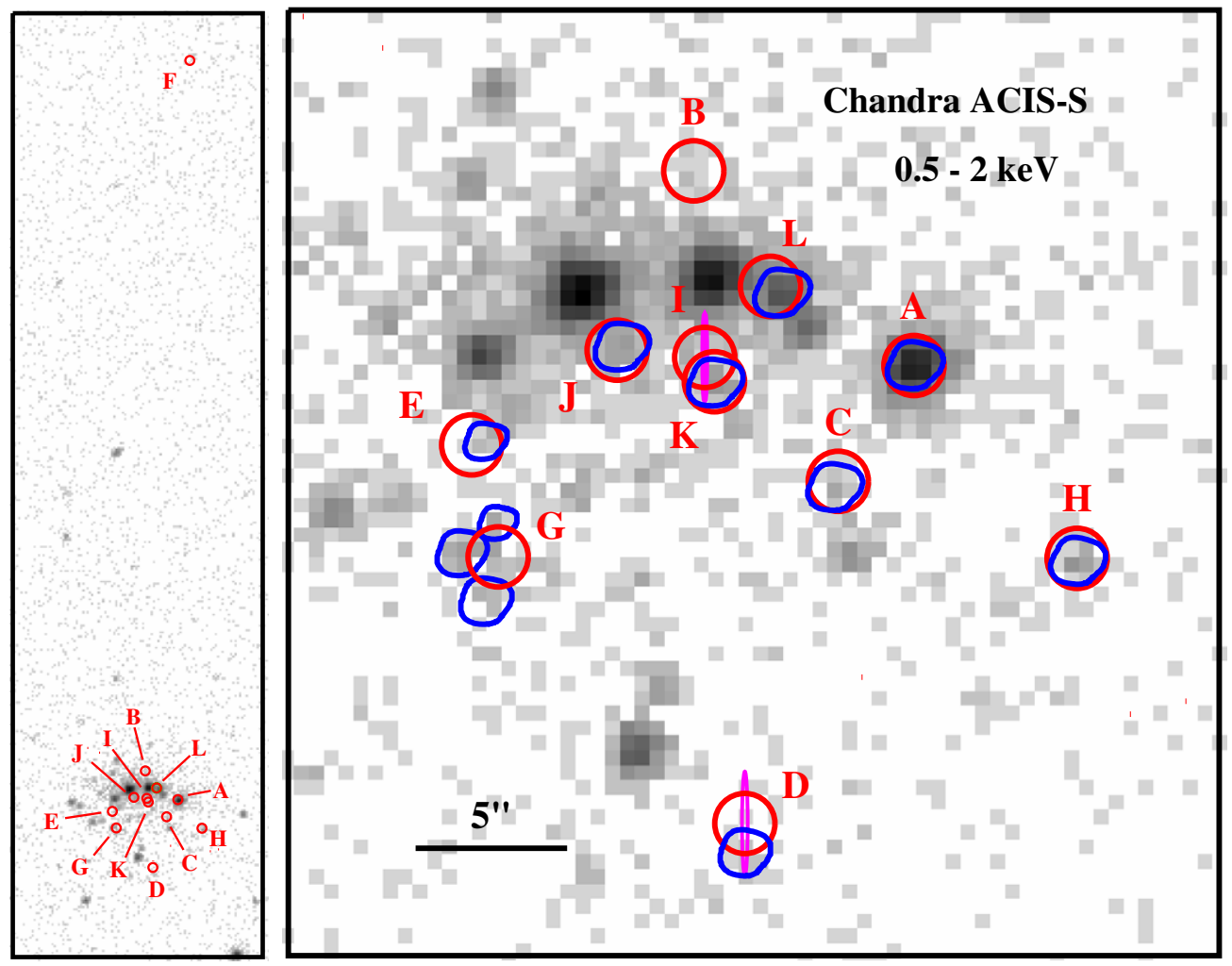

Figure 1. Left: Chandra ACIS-S co-added $0.5-2 \mathrm{keV}$ image of the globular cluster M28 with the positions of the 12 known radio pulsars in the cluster labeled. Right: the inner core of M28, with 1" radius red circles centered on the radio pulsar timing positions and blue polygons representing the extraction regions of the nearest $\mathrm{X}$-ray source to each pulsar, with the exception of pulsar $\mathrm{G}$ for which the nearest three sources are shown. The magenta ellipses show the TEMPO radio timing position uncertain for pulsars D and I, multiplied by a factor of two and five, respectively (see the text for details). For all other pulsars, the radio position uncertainties are much smaller ( $\left.\lesssim 0^{\prime} \cdot 1\right)$. The image gray scale corresponds to number of counts increasing logarithmically from 0 (white) to 2553 (black). North is up and east is to the left.

timing solution and thus a precise radio timing position (see $\mathrm{S}$. Bégin et al. 2011, in preparation for details). Thus, the error ellipse shown in Figure 1, taken to be five times the formal position uncertainty derived from a TEMPO fit with a $\chi_{v}^{2}$ over 7 , represents the current best estimate of the location of pulsar I, which may be subject to significant change. We note that the best timing fit of the eclipsing binary MSP $\mathrm{H}$ also has a large $\chi_{v}^{2}$, implying that the uncertainties in the position reported by TEMPO are not realistic estimates of the true errors. Fortunately, the recent identification of its optical counterpart by Pallanca et al. (2010) provides a much more reliable localization of $\mathrm{M} 28 \mathrm{H}$.

As evident from Figure 1 and Table 2, MSPs A, C, H, J, and $\mathrm{K}$ are found well within the $95 \%$ error circles of $\mathrm{X}$-ray sources, with differences between the $\mathrm{X}$-ray and radio positions of $\lesssim 0^{\prime}$.24. Thus, we deem the X-ray identifications of these pulsars as secure. For pulsar D, a single X-ray source falls within the radio timing error ellipse. As the source is found away from the crowded cluster core, it is most likely the X-ray counterpart to the pulsar. Pulsar F is located just 0.04 outside of $r_{95 \%}$ of the sole X-ray source in the vicinity, suggesting that the two are related. It is likely that the small positional discrepancy is due to a slight rotational offset between the X-ray and radio astrometric frames. However, as pulsar A is the only bright $\mathrm{X}$-ray source with a radio counterpart in this field, it is not possible to unambiguously correct for this effect. Pulsar E is found 0.12 outside of the $95 \%$ error circle of the nearest X-ray source. As this MSP is located in the crowded cluster core, this association is less certain. Although faint emission is coincident with the position of MSP B, the source detection algorithms fail to identify an X-ray counterpart to this pulsar. The detection of MSP I is rendered difficult by its position between MSP K and one of the brightest sources in the cluster (23 in Becker et al. 2003). The centroid of the X-ray counterpart to pulsar $\mathrm{K}$ is nearly coincident with its radio position $\left(\left|P_{R}-P_{X}\right|=\right.$ 0 .'03), implying that pulsar I is either significantly fainter than $\mathrm{K}$ or is not at the location derived from radio timing. MSP L is nearly coincident with a moderately luminous X-ray source (22 in Becker et al. 2003). As discussed in Section 6, however, based on the properties of this pulsar as inferred from radio observations and the temporal behavior of the X-ray emission from source 22, the two objects are most likely not physically associated. For MSP G, three X-ray sources are found $\sim 1^{\prime \prime}$ away (see Figure 1), making the X-ray-radio association ambiguous as the X-ray photons coincident with its radio position may be due to collective emission from the three nearby X-ray sources. It is also difficult to reliably constrain the intrinsic pulsar flux as there is no appropriate region from which to extract a representative background. Consequently, in order to place a crude upper limit on the flux, we extracted source counts within $1^{\prime \prime}$ of the radio position.

Given the high X-ray source number density in the cluster core, it is important to investigate the possibility that X-ray sources coincide with most of the radio pulsar positions purely by chance (with the exception of M28 A, for which the radio$\mathrm{X}$-ray association is well established). We have applied systematic offsets in the range $0.5^{\prime \prime}-5^{\prime \prime}$ in all directions of the radio pulsar positions with respect to the X-ray data set. We find that any offset consistently results in two or less source matches. The same holds true for all angles of rotation of the radio 
Table 2

Net Counts and Luminosities for the Pulsars in M28

\begin{tabular}{lcccccc}
\hline \hline PSR J1824-2452 & $\begin{array}{c}\text { Soft }^{\mathrm{a}} \\
(0.3-1.5 \mathrm{keV})\end{array}$ & $\begin{array}{c}\text { Hard }^{\mathrm{a}} \\
(1.5-6 \mathrm{keV})\end{array}$ & $\begin{array}{c}L_{X} \mathrm{~b}^{\mathrm{b}} \\
(0.5-8 \mathrm{keV})\end{array}$ & $\begin{array}{c}r_{95 \%^{\mathrm{c}}} \\
\left(^{\prime \prime}\right)\end{array}$ & $\begin{array}{c}\left|P_{R}-P_{X}\right|^{\mathrm{d}} \\
\left({ }^{\prime \prime}\right)\end{array}$ & Detection \\
\hline $\mathrm{A}$ & 2106 & 3800 & 1147.0 & 0.28 & 0.0 & Yes \\
$\mathrm{B}$ & $\lesssim 11$ & $\lesssim 10$ & $\lesssim 2$ & $\ldots$ & $\ldots$ & No \\
$\mathrm{C}$ & 11 & 6 & 1.7 & 0.39 & 0.21 & Yes \\
$\mathrm{D}$ & 8 & 2 & 1.0 & 0.44 & 0.99 & Yes \\
$\mathrm{E}$ & 8 & 8 & 2.0 & 0.40 & 0.49 & $?$ \\
$\mathrm{~F}$ & 13 & 10 & 1.2 & 0.50 & 0.54 & Yes \\
$\mathrm{G}^{\mathrm{f}}$ & 27 & 6 & $<10.8$ & 0.33 & $1.19 ?$ & $?$ \\
$\mathrm{H}$ & 23 & 49 & 16.9 & 0.33 & 0.10 & Yes \\
$\mathrm{I}^{\mathrm{e}}$ & 0 & 0 & $\lesssim 1$ & $\ldots$ & $\ldots$ & No \\
$\mathrm{J}$ & 37 & 21 & 1.3 & 0.34 & 0.20 & Yes \\
$\mathrm{K}$ & 26 & 20 & 5.4 & 0.35 & 0.03 & Yes \\
$\mathrm{L}^{\mathrm{f}}$ & 350 & 536 & $<189.2$ & 0.30 & 0.43 & No \\
\hline
\end{tabular}

Notes.

${ }^{a}$ Background-subtracted net counts extracted from regions enclosing $\sim 90 \%$ of the total energy at $1.5 \mathrm{keV}$ from the nearest $\mathrm{X}$-ray source to each pulsar. For MSPs I and B, for which no X-ray counterpart is detected, the source counts were extracted from a circle of radius 1 1"7 centered on the radio timing position.

${ }^{\mathrm{b}}$ Estimated unabsorbed X-ray luminosity in units of $10^{30} \mathrm{erg} \mathrm{s}^{-1}$ in the $0.5-8 \mathrm{keV}$ band based on Cash statistic (Cash 1979) fits to the spectra with a power-law model for $N_{\mathrm{H}}=2.4 \times 10^{21} \mathrm{~cm}^{-2}$, and $D=5.5 \mathrm{kpc}$. For MSPs A, G, H, and L, the values were derived from spectral fitting using the $\chi^{2}$ statistic with $N_{\mathrm{H}}$ as a free parameter (see Table 3 ).

${ }^{c}$ Positional uncertainty (95\% confidence error circle radius) in arcseconds of the putative $\mathrm{X}$-ray counterpart to each radio MSP, determined using Equation (5) from Hong et al. (2005). For pulsars B and I, there are no plausible X-ray counterparts in the vicinity.

${ }^{d}$ Angular separation in arcseconds between the radio timing position of each MSP (from S. Bégin et al. 2011, in preparation) and the centroid of the nearest X-ray source. For pulsar A, the difference is zero as this pulsar was used to align the radio and $\mathrm{X}$-ray astrometric frames.

${ }^{\mathrm{e}}$ Emission strongly contaminated by neighboring bright source.

${ }^{\mathrm{f}}$ Possibly an unresolved blend of two or more unrelated sources.

Table 3

Spectral Fits for M28 MSPs A, G, H, and L

\begin{tabular}{lcllc}
\hline \hline MSP & $\mathbf{A}$ & \multicolumn{1}{c}{$\mathrm{G}^{\mathrm{a}}$} & \multicolumn{1}{c}{$\mathbf{H}$} & $\mathrm{L}^{\mathrm{a}}$ \\
\hline Power law & & & & \\
\hline$N_{\mathrm{H}}{ }^{\mathrm{b}}$ & $\mathbf{2 . 5 5}_{-\mathbf{0 . 1 2}}^{+\mathbf{0 . 1 3}}$ & $1.25_{-0.78}^{+0.60}$ & $\mathbf{2 . 1}_{-\mathbf{1 . 1}}^{+\mathbf{1 . 5}}$ & $3.2_{-0.3}^{+0.4}$ \\
$\Gamma$ & $\mathbf{1 . 2 2 6}_{-\mathbf{0 . 0 2 8}}^{+\mathbf{0 . 0 2 5}}$ & $2.12_{-0.25}^{+0.21}$ & $\mathbf{1 . 0 9}_{-\mathbf{0 . 2 9}}^{+\mathbf{0 . 2 8}}$ & $1.52_{-0.08}^{+0.07}$ \\
$F_{X}{ }^{\mathrm{c}}$ & $\mathbf{3 8 0}_{-\mathbf{9}}^{+\mathbf{3}}$ & $3.6_{-1.1}^{+0.6}$ & $\mathbf{4 . 8}_{-\mathbf{3 . 6}}^{+\mathbf{0 . 9}}$ & $55.4_{-5.0}^{+4.9}$ \\
$\chi_{v}^{2} /$ d.o.f. & $\mathbf{0 . 9 3 / 2 5 3}$ & $0.89 / 4$ & $\mathbf{0 . 8 7 / 3}$ & $0.82 / 54$ \\
\hline
\end{tabular}

Notes. The bold values are for those MSPs (A and H) that were unambiguously detected.

${ }^{a}$ Spectrum likely dominated by unrelated X-ray source(s).

${ }^{\mathrm{b}}$ Hydrogen column density along the line of sight in units of $10^{21} \mathrm{~cm}^{-2}$.

${ }^{c}$ Unabsorbed flux in units of $10^{-15} \mathrm{erg} \mathrm{cm}^{-2} \mathrm{~s}^{-1}$ in the $0.3-8 \mathrm{keV}$ band.

pulsar positions about the cluster center or about the position of pulsar A. This finding suggests that the majority of the radio$\mathrm{X}$-ray source matches are indeed genuine and are consistent with pulsars $\mathrm{L}$ and/or $\mathrm{G}$ being possible false matches.

Table 2 summarizes the background-subtracted X-ray net source counts extracted from polygonal regions that enclose $\sim 90 \%$ of the total energy at $1.5 \mathrm{keV}$ for the putative X-ray counterparts of the pulsars. For MSPs I and B, for which no $\mathrm{X}$-ray counterparts are detected, we extracted source counts from circles of radius 1".7 (enclosing 95\% of the energy at $1.5 \mathrm{keV}$ ) centered on the radio timing positions. The background event contribution was determined from 1".7 circles that are the same radial distance from source 23 (Becker et al. 2003) as the MSPs. In both cases, the net source counts are consistent with zero.

For the X-ray-faint pulsars (C, D, E, F, J, and K), we estimate the unabsorbed X-ray luminosity by fitting a power-law spectrum with $N_{H}=2.4 \times 10^{21} \mathrm{~cm}^{-2}$ using the Cash statistic (Cash 1979) in XSPEC. This particular spectral model was chosen because it reproduces the rough spectral shape of a twotemperature thermal model observed in nearby MSPs (Becker \& Aschenbach 2002; Zavlin 2006; Bogdanov \& Grindlay 2009) quite well for crude photon statistics, with spectral photon index in the range 2-3.5. In the cases of MSPs A, G, H, and L the luminosities were derived from the spectral fits discussed in Sections 4 and 5, although for $\mathrm{G}$ and $\mathrm{L}$ these values most probably do not correspond to the intrinsic pulsar values as a consequence of source confusion/blending.

We note that in the combined Chandra HRC-S data (totaling $90 \mathrm{ks}$ ), of the 12 pulsars only pulsar $\mathrm{A}$ is detected, owing to the $\sim 3$ times lower sensitivity at $0.5-3 \mathrm{keV}$ and the higher background of HRC compared to ACIS.

\section{PSR B1821-24}

PSR B1821-24 is the first MSP to be discovered in a globular cluster (Lyne et al. 1987). This solitary pulsar with $P=$ $3.05 \mathrm{~ms}, \dot{P} \equiv d P / d t=1.61 \times 10^{-18} \mathrm{~s} \mathrm{~s}^{-1}$, is $\sim 100$ times younger than most "recycled" MSPs, based on its characteristic age $\left(\tau_{c}=P / 2 \dot{P}=30 \mathrm{Myr}\right)$. It is also the most energetic MSP known, with spin-down luminosity $\dot{E}=2.2 \times$ $10^{36} \mathrm{erg} \mathrm{s}^{-1}$ and X-ray luminosity $L_{X} \approx 1.3 \times 10^{33} \mathrm{erg} \mathrm{s}^{-1}$ (phase integrated for $0.5-8 \mathrm{keV}$; see Becker et al. 2003), 2-3 orders of magnitude greater than typical MSPs. Only two other X-ray detected MSPs, PSRs B1937+21 and J0218+4232 (both field MSPs), have comparable values of $L_{X}$ (Cusumano et al. 2003; Webb et al. 2004).

Because of the bright nature of M28 A, 4\% of the X-ray photons from this source in the ACIS-S observations are 


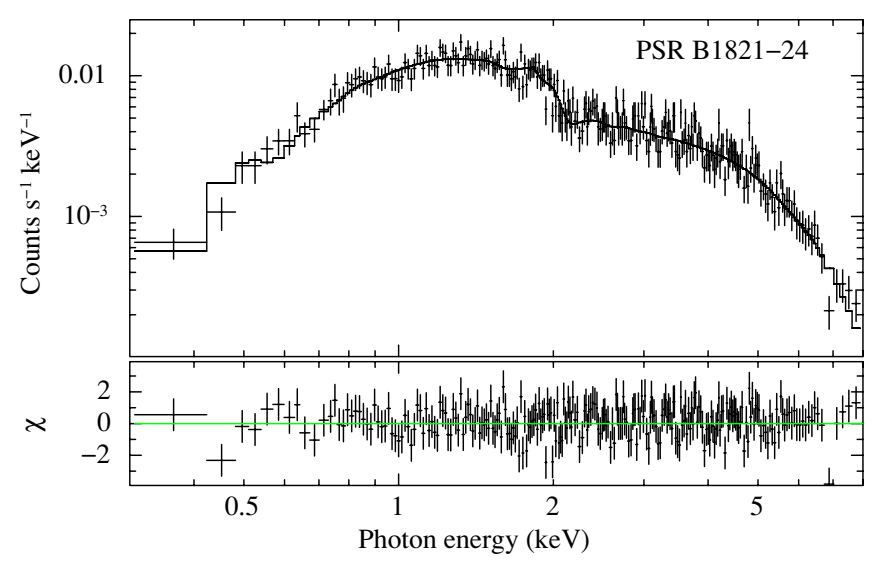

Figure 2. Phase-integrated Chandra ACIS-S X-ray spectrum of PSR B1821-24 fitted with an absorbed power-law spectrum. The lower panel shows the best-fit residuals. See the text and Table 3 for best-fit parameters.

(A color version of this figure is available in the online journal.)

expected to suffer from pileup (based on PIMMS ${ }^{15}$ ). Pileup occurs when two or more photons arrive within the same detector pixel within a single ACIS frame integration time and are registered as a single event. The detected energy of such an event is approximately equal to the sum of the energies of the individual photon events of which it is comprised. The result is a distortion of the intrinsic source spectrum (Davis 2001). To ascertain the severity of this effect, we have included the pileup model provided in XSPEC in our spectral fits. We find that pileup has negligible impact on the spectrum of PSR B1821-24, as the best-fit values of the model parameters with and without pileup are well within $1 \sigma$ of one another. Based on this, in what follows, we ignore the effect of pileup.

As expected, the phase-integrated X-ray spectrum of PSR B1821-24 is very well described by a pure absorbed power law (Figure 2). The best-fit parameters are $\Gamma=1.226_{-0.028}^{+0.025}$, $N_{\mathrm{H}}=\left(2.55_{-0.12}^{+0.13}\right) \times 10^{21} \mathrm{~cm}^{-2}$ with $\chi_{v}^{2}=0.93$ for $253 \mathrm{de}-$ grees of freedom. The uncertainties quoted are $1 \sigma$ for one interesting parameter. The unabsorbed energy flux is $F_{X}=$ $\left(3.80_{-0.09}^{+0.13}\right) \times 10^{-13} \mathrm{erg} \mathrm{s}^{-1}(0.3-8 \mathrm{keV})$, corresponding to a luminosity of $L_{X}=1.4 \times 10^{33} \Theta(D / 5.5 \mathrm{kpc})^{2} \mathrm{erg} \mathrm{s}^{-1}$, where $\Theta$ is the (unknown) portion of the sky covered by the X-ray emission beams.

By analogy with most MSPs, M28 A may have heated magnetic polar caps. As a result, low-level thermal emission could be present in the predominantly non-thermal spectrum of PSR B1821-24. Introducing either a blackbody or hydrogen atmosphere model (NSATMOS from McClintock et al. 2004) with temperatures in the range $(0.5-3) \times 10^{6} \mathrm{~K}$ yields acceptable fits, although given the high quality of the pure power-law fit, the addition of the thermal component is not warranted by the data (as confirmed by an $F$-test). This allows us to set a firm upper bound on any thermal component of $\sim 1 \%$ of the total energy flux in the $0.3-8 \mathrm{keV}$ range. The implied thermal luminosity of $\lesssim 10^{31} \mathrm{erg} \mathrm{s}^{-1}$ is comparable to most nearby MSPs (Zavlin 2006; Bogdanov \& Grindlay 2009) and those in 47 Tuc (Bogdanov et al. 2006). On the other hand, in some theoretical pulsar models (see, e.g., Harding \& Muslimov 2002), the energetics of the magnetosphere of PSR B1821-24 may not be conducive to the production of a substantial return current of particles to heat the polar cap surface so this pulsar may in fact have much cooler polar caps.

\footnotetext{
15 See http://cxc.harvard.edu/toolkit/pimms.jsp.
}

Becker et al. (2003) have reported intriguing but marginal evidence (at $98 \%$ confidence) for a broad emission feature centered at $3.3 \mathrm{keV}$, which they interpreted as cyclotron emission from the magnetosphere above the pulsar polar cap if the magnetic field strongly deviates from a centered dipole. We have reprocessed the archival ACIS-S data using the latest Chandra X-ray Center calibration products and find no evidence of this feature. As seen from Figure 2, the broad spectral feature is absent in the total ACIS-S spectrum as well. Therefore, we attribute the deviation from a pure power law of the PSR B1821-24 spectrum found by Becker et al. (2003) to an unmodeled instrumental feature that has since been incorporated into the telescope calibration model.

\subsection{The Unpulsed X-ray Emission from PSR B1821-24}

The bulk of X-rays from PSR B1821-24 ( 85\%) are emitted in two very narrow pulses (Rutledge et al. 2004), indicative of highly beamed, non-thermal radiation from the pulsar magnetosphere, in stark contrast with the broad, soft thermal pulsations of most MSPs (Zavlin 2006; Bogdanov et al. 2006; Cameron et al. 2007; Bogdanov \& Grindlay 2009). Surprisingly, Rutledge et al. (2004) have found an appreciable unpulsed component, contributing $\sim 15 \%$ of total photon flux.

Using the two HRC-S timing observations of PSR B1821-24, we can place constraints on the origin of the puzzling unpulsed emission. To this end, we combined the pulse profiles from the two HRC data sets as follows. We first folded the barycentered arrival times of the events extracted from a $1^{\prime \prime}$ region centered on the radio pulsar in $\mathrm{TEMPO}^{16}$ using the timing ephemeris from Ray et al. (2008) for each data set separately. Due to the $\sim 3.5$ year gap between the two HRC-S observations, it is not possible to phase connect the two data sets accurately using the available radio timing ephemerides. Thus, to determine the relative phase misalignment, we cross-correlated the folded pulse profiles from both observations. The individual and combined pulse profiles are shown in Figure 3. The summed HRC-S pulse profile yields 706 photons, a 1.8-fold improvement in photon statistics over the data from Rutledge et al. (2004). Of these, $16 \pm 0.4$ photons are due to background. As in Rutledge et al. (2004), the unpulsed (DC) level in the summed profile was determined by considering the emission \pm 0.15 in phase away from the peaks of the two pulses to minimize the contribution from the pulsed component. After subtracting the background contribution, we find that $17.5 \% \pm 4 \%$ of the source photons are unpulsed. Varying the choice of phase intervals used to determine the unpulsed level results in a spread of $\pm 1 \%$ around the nominal value.

Although the HRC has poor intrinsic energy resolution, in principle, it is still possible to determine any gross difference between the spectra of the pulsed and unpulsed emission using hardness ratios. ${ }^{17}$ The hardness ratios, however, do not show any substantial differences in the spectra of the pulsed and unpulsed portions, implying that the unpulsed emission is not drastically softer or harder than the pulsed emission. Nonetheless, the highquality phase-integrated ACIS-S continuum provides stringent constraints on the spectral properties of the unpulsed emission, in particular, if it is of a thermal nature or not. The best-fit absorbed power-law model derived from the ACIS-S spectrum, convolved with the HRC-S effective area curve, reveals that the bulk of photons from PSR B1821-24 in the HRC-S data sets

\footnotetext{
16 Available at http://tempo.sourceforge.net.

17 See http://cxc.harvard.edu/proposer/POG/html/chap7.html for details.
} 


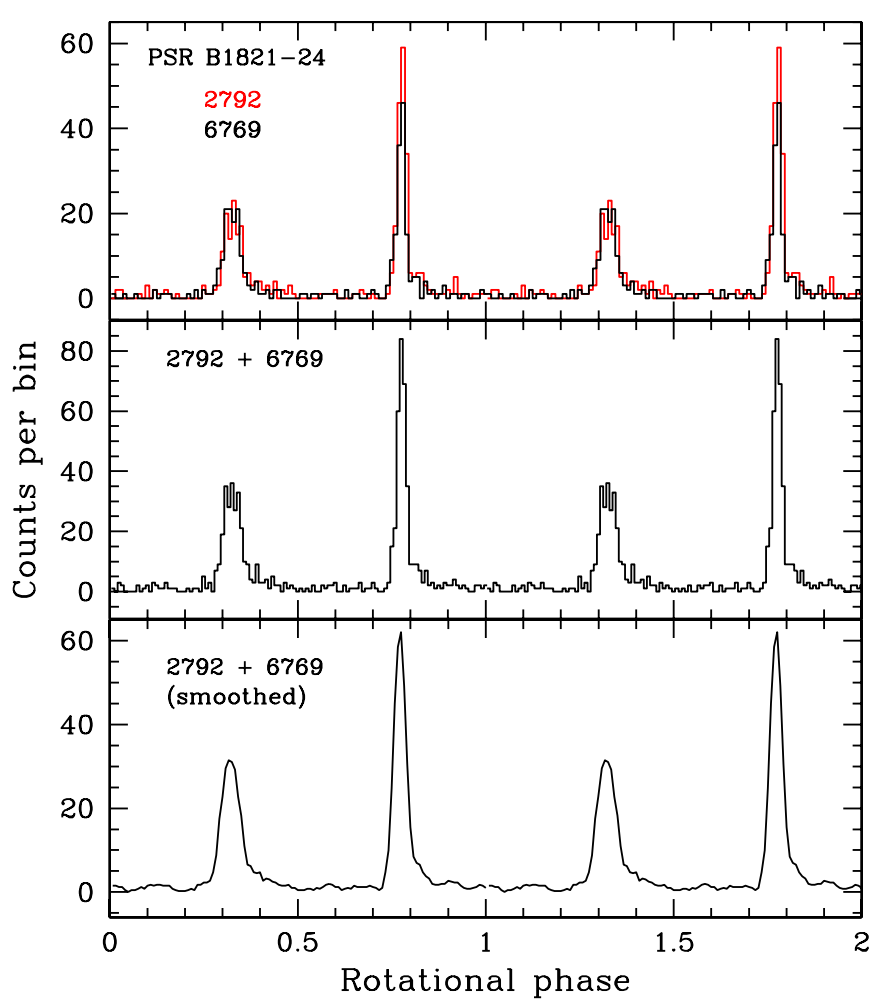

Figure 3. Chandra HRC-S pulse profiles of PSR B1821-24 from ObsIDs 2797 and 6769 (top panel) and the summed pulse profile (middle panel). The bottom panel shows the summed profile smoothed using a four-point moving average. Note the asymmetry of the wings of the two pulses. The choice of phase zero is arbitrary. Two cycles are shown for clarity.

(An animation of this figure is available in the online journal.)

are from the $0.4-2 \mathrm{keV}$ range. We fitted the ACIS-S spectrum with additional thermal components to determine their photon flux contribution in this energy range. Converting the resulting values to the equivalent HRC-S count rates indicates that any thermal emission can contribute no more than $\sim 6 \%$ of the total number of photons from M28 A in the HRC-S observations. This leaves at least $\sim 7 \%-15 \%$ of the photons unaccounted for.

It is interesting to speculate on the origin of this excess unpulsed emission. In many young, energetic pulsars, with the Crab pulsar being the most notable example (Kirsch et al. 2006), the high-energy non-thermal pulse profiles often exhibit a "bridge" of emission connecting two pulses, suggesting that the two pulses originate from the same emission cone. However, for PSR B1821-24 the count rates in the regions between the two pulses are consistent with being identical. The apparent lack of excess of photons in one of the regions implies that bridge emission is not responsible for the seemingly unpulsed photons. It should be noted however that both pulses exhibit tails of emission from their trailing edges (see bottom panel of Figure 4), which could plausibly extend to cover most of the rotational period. Indeed, certain high-energy emission models of pulsars, such as the two-pole caustic model, predict lowlevel non-thermal magnetospheric emission spanning the entire period of rotation (Venter et al. 2010).

A plausible alternative origin for the unpulsed photons is synchrotron emission from an unresolved wind nebula surrounding this rather energetic pulsar. By analogy with pulsars with comparable spin-down luminosities (see Kargaltsev \& Pavlov 2008, for a review), the expected angular size of a wind nebula sur-
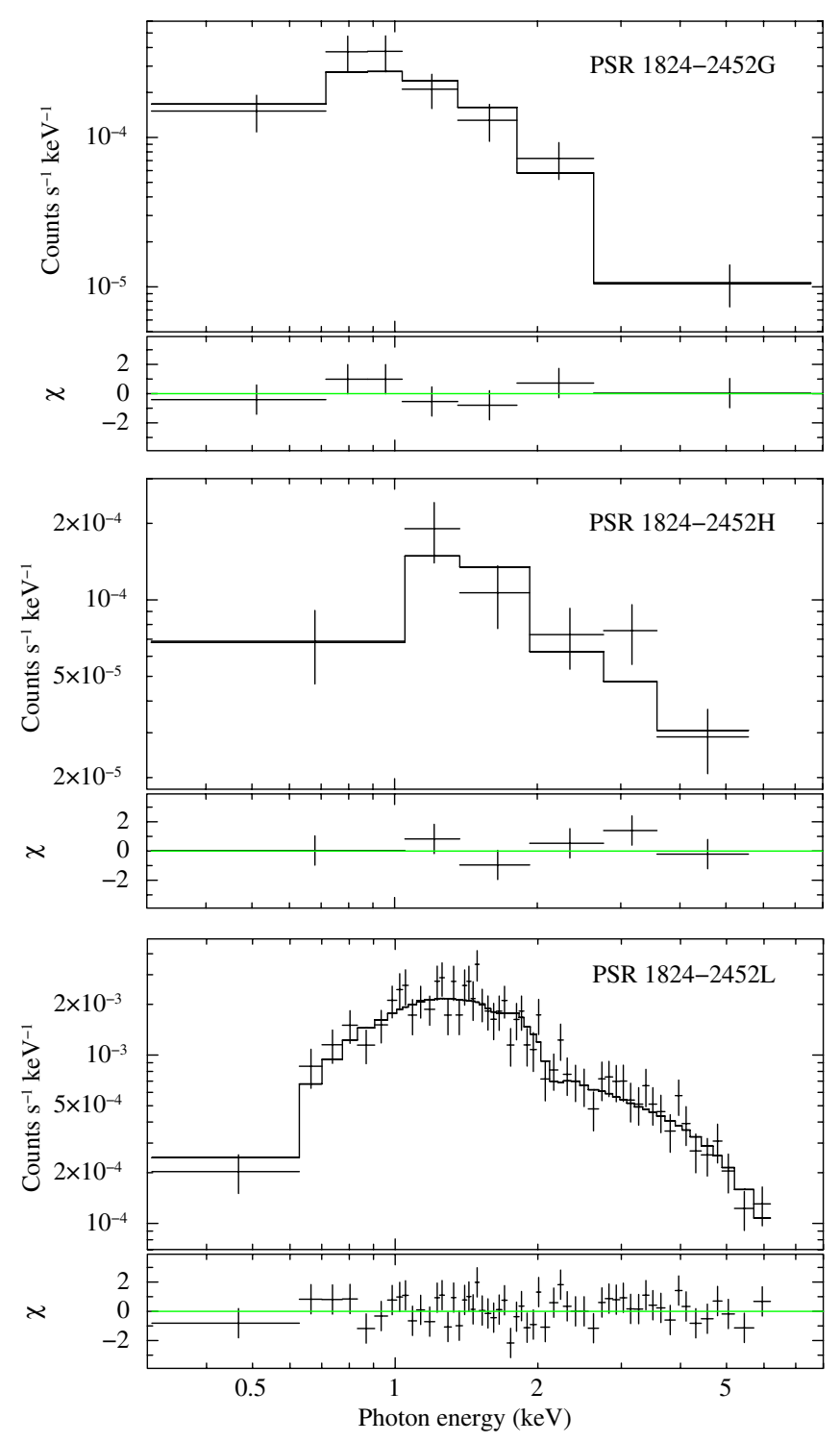

Figure 4. X-ray spectra of PSR J1824-2452H and of the X-ray sources nearest to PSRs J1824-2452G and L, fitted with power-law spectra. The bottom of each panel shows the best-fit residuals. See Table 3 for best-fit parameters.

rounding pulsar M28 A, assuming a distance of $5.5 \mathrm{kpc}$, would be $\sim 1^{\prime \prime}$. Such a nebula would be difficult to resolve, especially considering the source crowding in the cluster core. If the unpulsed emission arises solely due to the wind nebula, its implied $\mathrm{X}$-ray luminosity is $L_{\mathrm{PWN}} \sim 10^{32} \mathrm{erg} \mathrm{s}^{-1}$, similar to what is observed for other energetically comparable pulsars (see in particular Tables 1 and 2 in Kargaltsev \& Pavlov 2008).

Conceivably, the unpulsed emission could be caused by smallangle scattering of X-rays passing through dust grains in the interstellar medium (Smith et al. 2002). The additional path length of the scattered X-rays would induce a time delay in the photon time of arrival, causing them to become out of phase relative to the unscattered photons and thus form an unpulsed component. This effect could also account for the trailing tails of the two pulses. The location of M28 in the Galactic disk ( $l=7.8, b=5.6$ ) suggests that dust scattering is not negligible. We estimate the optical depth to scattering using the empirical relation $\tau_{\text {scat }, 1 \mathrm{keV}}=A_{V}(0.079 \pm 0.003)-(0.052 \pm 0.019)$ (Predehl \& Schmitt 1995), where $A_{V}$ is the visual interstellar 
extinction. For $A_{V} \sim 1.4 \mathrm{mag}$ toward M28 (Davidge 1996), this yields $\tau \sim 0.05$ implying that $I / I_{o}=e^{-\tau} \sim 5 \%$ of the $\mathrm{X}$-ray photons undergo scattering.

Finally, it is possible that at least a portion of the unpulsed component from PSR B1821-24 is due to one or more fainter, unrelated X-ray sources within $\sim 1^{\prime \prime}$ of PSR B1821-24. However, using wavdetect we find that the centroid of the unpulsed emission is offset by only $00^{\prime} .07$ from the centroid of the total emission, suggesting that source contamination is unlikely.

\section{PSR J1824-2452H}

Apart from PSR B1821-24, the binary PSR J1824-2452H is the only other unambiguously detected MSP with sufficient counts for a spectral fitting analysis. PSR J1824-2458H is in a $10.4 \mathrm{hr}$ circular orbit around what appears to be a non-degenerate star (Pallanca et al. 2010) and exhibits highly variable and irregular radio eclipses (S. Bégin et al. 2011, in preparation). This atypical MSP binary system is thus likely the result of a past dynamical binary exchange encounter in the dense cluster core (Camilo \& Rasio 2005), like PSR J1740-5340 in NGC 6397 (D'Amico et al. 2001; Grindlay et al. 2002; Bogdanov et al. 2010; Huang \& Becker 2010) and PSR J00247204W in 47 Tuc (Camilo et al. 2000; Edmonds et al. 2002; Bogdanov et al. 2005). Alternatively, it may have just emerged from the low-mass X-ray binary phase with the MSP reactivating as a rotation-powered pulsar, as is also possibly the case for PSR J1023+0038 (see Archibald et al. 2009). The X-ray properties of PSR J1824-2452H are thus of particular interest as these systems show unusual X-ray properties (Stappers et al. 2003; Bogdanov et al. 2005, 2010; Archibald et al. 2010). We fitted the spectrum of this MSP with a power-law model (middle panel of Figure 4), yielding $N_{\mathrm{H}}=\left(2.1_{-1.1}^{+1.5}\right) \times 10^{21} \mathrm{~cm}^{-2}$, $\Gamma=1.09_{-0.29}^{+0.28}$, and unabsorbed flux of $F_{X}=\left(4.8_{-3.6}^{+0.9}\right) \times 10^{-15}$ $(0.3-8 \mathrm{keV})$, with $\chi_{v}^{2}=0.87$ for 5 degrees of freedom. The nominal best-fit flux translates to a luminosity of $L_{X}=1.7 \times$ $10^{31} \mathrm{erg} \mathrm{s}^{-1}$ for a $5.5 \mathrm{kpc}$ distance. Despite the limited photon statistics, the spectrum is poorly reproduced by a singletemperature thermal model. Applying models with two or more components offers no meaningful constraints on any model parameters.

For this binary MSP, we can also investigate any large amplitude X-ray variability as a function of orbital phase, which could potentially provide clues regarding the origin of the $\mathrm{X}$-ray emission. We have used the radio timing ephemeris of PSR J1824-2452H (S. Bégin et al. 2011, in preparation) to fold the X-ray data set at the binary period. The resulting light curve (Figure 5) shows compelling evidence for variability. For four and five phase bins over the binary orbit, the variability is significant at a $4 \sigma$ and $2.7 \sigma$ level, respectively, based on a $\chi^{2}$ test. A Kuiper test (Paltani 2004) on the unbinned light curve, weighted to account for the non-uniform exposure across the orbit, indicates a $5.8 \times 10^{-4}(3.2 \sigma)$ probability that photons being drawn from a constant distribution would exhibit this level of non-uniformity. It is interesting to note that the minimum around $\phi=0.25$ in the X-ray light curve is roughly coincident with the position of the radio eclipse. Moreover, the rough shape of the light curve is remarkably similar to those of the analogous binaries PSRs J0024-7204W (Bogdanov et al. 2005), J1740-5340 (Bogdanov et al. 2010), and J1023+0038 (Archibald et al. 2010). Thus, the peculiar nature of the PSR J1824-2452H binary system, the implied X-ray luminosity, relatively hard spectrum compared to most MSPs observed in

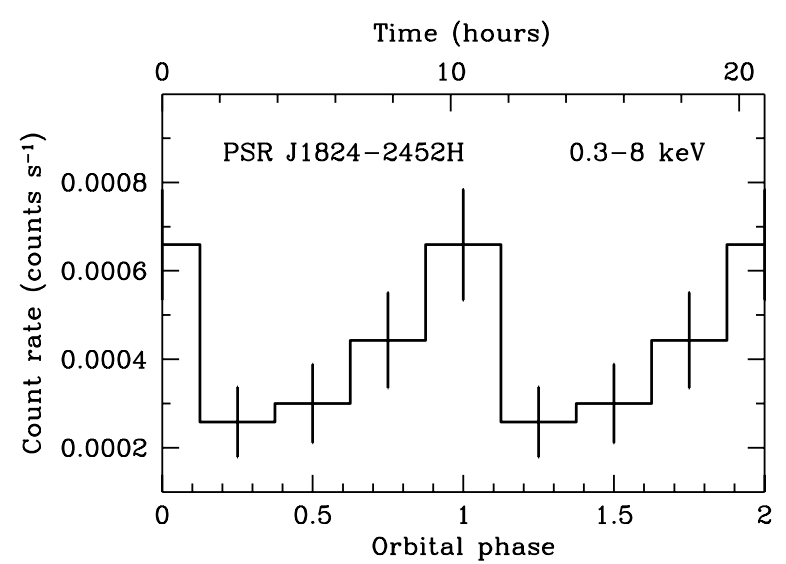

Figure 5. X-ray light curve of PSR J1824-2452H folded at the 10.4 hr binary period of the pulsar. The orbital phase is defined based on the radio pulsar timing convention in which superior conjunction occurs at $\phi=0.25$. Two orbital cycles are shown for clarity.

$\mathrm{X}$-rays, and the suggested variability at the binary period point to an intrabinary shock origin of the X-rays associated with this object. The minimum in the X-ray light curve at $\phi \approx 0.25$ can be plausibly interpreted as a geometric occultation (either partial or total) of the shock by the secondary star. Alternatively, it could arise due to relativistic beaming of the X-rays from the radiating particles in the shock. Due to the limited photon statistics and the number of orbits covered $(\sim 6)$, it is difficult to establish whether the flux modulation is truly periodic and stable over many orbits. As seen in PSR J0024-7204W (Bogdanov et al. 2005; Cameron et al. 2007), the orbital variation of the X-ray flux may actually evolve on timescales spanning from months to years.

\section{OTHER MSPs}

The $\sim 10$ times larger absorbing column toward M28 compared to 47 Tuc and $\sim 1.2$ times greater distance make it more difficult to study soft X-ray sources such as thermal MSPs (Bogdanov et al. 2006) in a comparable exposure. As a result, most of the detected pulsars in M28 do not have adequate photon statistics for detailed spectral analyses. Nonetheless, we can still constrain the origin of the observed X-rays by considering the summed spectrum of pulsars B, C, D, E, $\mathrm{F}$, J, and $\mathrm{K}$ (shown in Figure 6). Indeed, the combined X-ray continuum of these seven pulsars is poorly described by a singletemperature thermal spectrum, while a pure power-law model requires $N_{\mathrm{H}}>4 \times 10^{21} \mathrm{~cm}^{-2}$ for an acceptable fit, substantially higher than the value suggested by the fit to the spectrum of PSR B1821-24. A composite blackbody plus power-law model for $N=2.4 \times 10^{21} \mathrm{~cm}^{-2}$ gives an acceptable fit with $k T=0.26 \pm 0.03 \mathrm{keV}, R_{\text {eff }}=0.19_{-0.13}^{+0.11} \mathrm{~km}, \Gamma=0.8_{-1.5}^{+0.9}$, and $\chi_{v}^{2}=0.86$ for 10 degrees of freedom. Note that a power-law component does not necessarily imply non-thermal emission since the superposition of several multi-temperature thermal MSP spectra can produce this spectral shape for limited photon statistics (Zavlin 2006; Bogdanov \& Grindlay 2009). The derived unabsorbed flux of $7.5 \times 10^{-15} \mathrm{erg} \mathrm{cm}^{-2} \mathrm{~s}^{-1}(0.3-8 \mathrm{keV})$ implies a mean X-ray luminosity of $3.9 \times 10^{30} \mathrm{erg} \mathrm{s}^{-1}$ for the seven pulsars or $4.5 \times 10^{30} \mathrm{erg} \mathrm{s}^{-1}$ if we exclude pulsar B. For comparison, the mean unabsorbed X-ray luminosity of the 11 thermal MSPs in 47 Tuc with unconfused and uncontaminated spectra is $\sim 3.2 \times 10^{30} \mathrm{erg} \mathrm{s}^{-1}$ (Bogdanov et al. 2006). This 


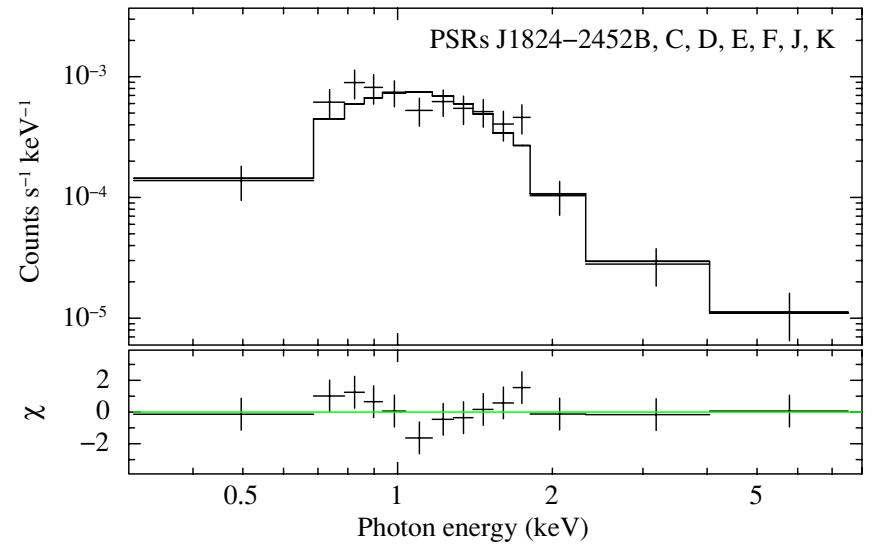

Figure 6. Summed X-ray spectrum of PSRs J1824-2452B, C, D, E, F, J, and $\mathrm{K}$ fitted with a blackbody plus power-law model. The bottom panels show the best-fit residuals. See the text for best-fit parameters.

(A color version of this figure is available in the online journal.)

suggests that the MSP populations of the two clusters are quite similar in terms of X-ray properties.

The non-detection of pulsars B and I implies that these pulsars may be below the sensitivity of the total ACIS-S exposure, with $L_{X} \lesssim 1 \times 10^{30} \mathrm{erg} \mathrm{s}^{-1}(0.3-8 \mathrm{keV})$, perhaps similar to the unusually sub-luminous PSR B1257+12 with $L_{X} \sim 2.5 \times$ $10^{29} \mathrm{erg} \mathrm{s}^{-1}$ in the same energy band (Pavlov et al. 2007). The low luminosity of the non-recycled pulsar D is similar to those of comparably old nearby pulsars (see Kargaltsev et al. 2006).

For pulsars $\mathrm{G}$ and $\mathrm{L}$, there are sufficient counts within $1^{\prime \prime}$ to conduct spectral fitting and search for variability, although as noted in Section 3, the association of this emission with the pulsars is questionable. For both sources, the spectra are well described by an absorbed power-law model (see Table 3 and Figure 4). In the case of pulsar G, there is no statistically significant variability. As for PSR J1824-2452L, the X-rays extracted from within $1^{\prime \prime}$ of the radio timing position exhibit variability on timescales of order hours. In addition, there is a substantial increase (a factor of $\sim 3$ ) in the count rate from the three 2002 ACIS-S observations to the two observations in 2008. In principle, such large-amplitude variability from an MSP binary could arise due to interaction of the pulsar wind with gas from the companion star. However, this MSP is not unusual and exhibits no radio eclipses, which are associated with intrabinary shocks in all known cases. Therefore, the variable $\mathrm{X}$-rays are most probably from source 22 from Becker et al. (2003), which is not related to the pulsar.

\section{CONCLUSION}

We have presented deep Chandra observations of the sample of known pulsars in the globular cluster M28. Seven of the 12 known pulsars in the cluster are securely detected (A, C, D, $\mathrm{F}, \mathrm{H}, \mathrm{J}$, and $\mathrm{K}$ ), two are possibly detected (E and $\mathrm{G})$, while three are not detected (B, L, and I). Most of the MSPs firmly or possibly detected appear to be relatively faint and soft sources most likely due to thermal polar cap emission. In this sense, they appear to be akin to the majority of "recycled" MSPs in 47 Tuc (Bogdanov et al. 2006) and the field of the Galaxy (Zavlin 2006; Bogdanov \& Grindlay 2009), with soft spectra and $L_{X} \sim 4 \times 10^{30}-10^{31} \mathrm{erg} \mathrm{s}^{-1}(0.3-8 \mathrm{keV})$. This provides further evidence that in typical MSPs, both in globular clusters and the field of the Galaxy, the X-ray emission is primarily of a thermal nature. As shown by Pavlov \& Zavlin (1997), Zavlin \& Pavlov (1998), Bogdanov et al. (2007), and Bogdanov et al. (2008), realistic modeling of the X-ray spectra and pulsations of these objects may potentially allow stringent constraints on the neutron star equation of state through measurement of the massto-radius ratio of these stars. As such, MSPs warrant further investigation in the X-ray band.

The most detailed X-ray spectrum to date of the energetic PSR B1821-24 is found to be well described by a pure power law with $\Gamma=1.2$, with no requirements for additional components. We set a limit on any thermal X-ray emission from the MSP of $\lesssim 10^{31} \mathrm{erg} \mathrm{s}^{-1}$ for $0.3-8 \mathrm{keV}$. The greatly improved ACIS-S spectrum of this pulsar shows no evidence for the previously reported spectral feature at $\sim 3.3 \mathrm{keV}$. A re-examination of the X-ray pulse profile of PSR B1821-24 reveals that the observed unpulsed emission is unlikely to be of thermal origin and is due to either low-level non-thermal magnetospheric emission, unresolved nebular emission, and/or dust scattering of the pulsed X-rays.

PSR J1824-2452H appears to share common X-ray characteristics (namely, variable and hard non-thermal emission with $L_{X} \sim 10^{31} \mathrm{erg} \mathrm{s}^{-1}$ ) with PSRs J0024-7204W, J1023+0039, and J1740-5340. Presumably, in these peculiar binary systems the X-rays originate from an intrabinary (synchrotron) X-ray emitting shock formed by interaction of the MSP wind and matter from the secondary star (Arons \& Tavani 1993). Furthermore, this class of objects exhibits remarkably similar X-ray and optical properties to the accreting X-ray MSPs in quiescence (Campana et al. 2004; Heinke et al. 2007, 2009) and thus can potentially offer vital clues into the little understood activation mechanism for rotation-powered MSPs.

We thank Z. Medin, A. Harding, and A. Archibald for useful discussions and the referee F. Camilo for comments. The work presented was funded in part by NASA Chandra grant GO89072X awarded through the Harvard College Observatory. S.B. is supported in part by a CIFAR Junior Fellowship. C.O.H. and I.H.S. are supported by NSERC. This research has made use of the NASA Astrophysics Data System (ADS) and software provided by the Chandra X-ray Center (CXC) in the application package CIAO.

Facility: $\mathrm{CXO}$

\section{REFERENCES}

Archibald, A. M., Kaspi, V. M., Bogdanov, S., Hessels, J. W. T., Stairs, I. H., Ransom, S. M., McLaughlin, M. A., \& Lorimer, D. 2010, ApJ, 722, 88 Archibald, A. M., et al. 2009, Science, 324, 1411

Arons, J., \& Tavani, M. 1993, ApJ, 403, 249

Bassa, C. G., et al. 2004, ApJ, 609, 755

Becker, W., \& Aschenbach, B. 2002, in Proc. 270 WE-Heraeus Seminar on Neutron Stars, Pulsars, and Supernova Remnants, ed. W. Becker, H. Lech, \& J. Trümper (Garching bei München: Max-Planck Inst. für Extraterr. Physik), 64

Becker, W., et al. 2003, ApJ, 594, 798

Bégin, S. 2006, Master's Thesis, Faculty of Physics, Univ. British Columbia Bogdanov, S., \& Grindlay, J. E. 2009, ApJ, 703, 1557

Bogdanov, S., Grindlay, J. E., Heinke, C. O., Camilo, F., Freire, P. C. C., \& Becker, W. 2006, ApJ, 646, 1104

Bogdanov, S., Grindlay, J. E., \& Rybicki, G. B. 2008, ApJ, 689, 407

Bogdanov, S., Grindlay, J. E., \& van den Berg, M. 2005, ApJ, 630, 1029

Bogdanov, S., Rybicki, G. B., \& Grindlay, J. E. 2007, ApJ, 670, 668

Bogdanov, S., van den Berg, M., Heinke, C. O., Cohn, H. N., Lugger, P. M., \& Grindlay, J. E. 2010, ApJ, 709, 241

Broos, P. S., Townsley, L. K., Feigelson, E. D., Getman, K. V., Bauer, F. E., \& Garmire, G. P. 2010, ApJ, 714, 1582 
Cameron, P. B., Rutledge, R. E., Camilo, F., Bildsten, L., Ransom, S. M., \& Kulkarni, S. R. 2007, ApJ, 660, 587

Camilo, F., Lorimer, D. R., Freire, P., Lyne, A. G., \& Manchester, R. N. 2000, ApJ, 535, 975

Camilo, F., \& Rasio, F. A. 2005, in ASP Conf. Ser. 328, Binary Radio Pulsars, ed. F. A. Rasio \& I. H. Stairs (San Francisco, CA: ASP), 147

Campana, S., et al. 2004, ApJ, 614, L49

Cash, W. 1979, ApJ, 228, 939

Cusumano, G., et al. 2003, A\&A, 410, L9

D’Amico, N., Lyne, A. G., Manchester, R. N., Possenti, A., \& Camilo, F. 2001, ApJ, 548, L171

D’Amico, N., Possenti, A., Fici, L., Manchester, R. N., Lyne, A. G., Camilo, F., \& Sarkissian, J. 2002, ApJ, 570, L89

Damiani, F., Maggio, A., Micela, G., \& Sciortino, S. 1997a, ApJ, 483, 350

Damiani, F., Maggio, A., Micela, G., \& Sciortino, S. 1997b, ApJ, 483, 370

Davidge, T. J. 1996, ApJ, 468, 641

Davis, J. E. 2001, ApJ, 562, 575

Edmonds, P. D., Gilliland, R. L., Camilo, F., Heinke, C. O., \& Grindlay, J. E. 2002, ApJ, 579, 741

Elsner, R. F., et al. 2008, ApJ, 687, 1019

Freire, P. C., Camilo, F., Kramer, M., Lorimer, D. R., Lyne, A. G., Manchester, R. N., \& D' Amico, N. 2003, MNRAS, 340, 1359

Grindlay, J. E., Camilo, F., Heinke, C. O., Edmonds, P. D., Cohn, H., \& Lugger, P. 2002, ApJ, 581, 470

Harding, A. K., \& Muslimov, A. G. 2002, ApJ, 568, 862

Harris, W. E. 1996, AJ, 112, 1487

Heinke, C. O., Jonker, P. G., Wijnands, R., Deloye, C. J., \& Taam, R. E. 2009, ApJ, 691, 1035

Heinke, C. O., Jonker, P. G., Wijnands, R., \& Taam, R. E. 2007, ApJ, 660, 1424

Heinke, C. O., Wijnands, R., Cohn, H. N., Lugger, P. M., Grindlay, J. E., Pooley, D., \& Lewin, W. H. G. 2006, ApJ, 651, 1098
Hong, J., van den Berg, M., Schlegel, E. M., Grindlay, J. E., Koenig, X., Laycock, S., \& Zhao, P. 2005, ApJ, 635, 907

Huang, R. H. H., \& Becker, W. 2010, A\&A, 510, 67

Kargaltsev, O., \& Pavlov, G. G. 2008, in AIP Conf. Proc. 983, 40 Years of Pulsars: Millisecond Pulsars, Magnetars and More, ed. C. Bassa et al. (Melville, NY: AIP), 171

Kargaltsev, O., Pavlov, G. G., \& Garmire, G. P. 2006, ApJ, 636, 406

Kirsch, M. G. G., et al. 2006, A\&A, 453, 173

Lyne, A. G., Brinklow, A., Middleditch, J., Kulkarni, S. R., \& Backer, D. C. 1987, Nature, 328, 399

McClintock, J. E., Narayan, R., \& Rybicki, G. B. 2004, ApJ, 615, 402

Pallanca, C., et al. 2010, ApJ, 725, 1165

Paltani, S. 2004, A\&A, 420, 789

Pavlov, G. G., Kargaltsev, O., Garmire, G. P., \& Wolszczan, A. 2007, ApJ, 664, 1072

Pavlov, G. G., \& Zavlin, V. E. 1997, ApJ, 490, L91

Predehl, P., \& Schmitt, J. H. M. M. 1995, A\&A, 293, 889

Ransom, S. M., Hessels, J. W. T., Stairs, I. H., Freire, P. C. C., Camilo, F., Kaspi, V. M., \& Kaplan, D. L. 2005, Science, 307, 892

Ray, P. S., Wolff, M. T., Demorest, P., Cognard, I., Backer, D. C., \& Wood, K. S. 2008, in AIP Conf. Proc. 983, 40 Years of Pulsars: Millisecond Pulsars, Magnetars and More, ed. C. Bassa et al. (Melville, NY: AIP), 157

Rutledge, R. E., Fox, D. W., Kulkarni, S. R., Jacoby, B. A., Cognard, I., Backer, D. C., \& Murray, S. S. 2004, ApJ, 613, 522

Shawl, S. J., \& White, R. E. 1986, AJ, 91, 312

Smith, R. K., Edgar, R. J., \& Shafer, R. A. 2002, ApJ, 581, 562

Stappers, B. W., Gaensler, B. M., Kaspi, V. M., van der Klis, M., \& Lewin, W. H. G. 2003, Science, 299, 1372

Venter, C., Harding, A. K., \& Guillemot, L. 2010, ApJ, 707, 800

Webb, N. A., Olive, J.-F., \& Barret, D. 2004, A\&A, 417, 181

Zavlin, V. E. 2006, ApJ, 638, 951

Zavlin, V. E., \& Pavlov, G. G. 1998, A\&A, 329, 583 